\title{
The braking indices in pulsar emission models
}

\author{
F. $\mathrm{Wu}^{1,2}$, R. X. $\mathrm{Xu}^{2}$, and J. Gil ${ }^{3}$ \\ 1 National Astronomical Observatories, Chinese Academy of Sciences, 20 Datun Road, Chaoyang, Beijing 100012, PR China \\ 2 School of Physics, Peking University, Beijing 100871, PR China \\ ${ }^{3}$ Institute of Astronomy, University of Zielona Góra, Lubuska 2, 65-265 Zielona Góra, Poland \\ Received 2 June 2003 / Accepted 30 June 2003

\begin{abstract}
Using the method proposed in a previous paper, we calculate pulsar braking indices in the models with torque contributions from both inner and outer accelerating regions, assuming that the interaction between them is negligible. We suggest that it is likely that the inverse Compton scattering induced polar vacuum gap and the outer gap coexist in the pulsar magnetosphere. We include the new near threshold vacuum gap models with curvature-radiation and inverse Compton scattering induced cascades, respectively; and find that these models can well reproduce the measured values of the braking indices.
\end{abstract}

Key words. stars: pulsars: general - radiation mechanisms: non-thermal

\section{Introduction}

Why do pulsars spin down? Although it is generally accepted that pulsars brake due to magnetodipole radiation, this simple model is questioned by the fact that the observed braking index $n \equiv \Omega \ddot{\Omega} / \dot{\Omega}^{2}$ ( $\Omega$ is the angular velocity of rotation) is smaller than 3. For the Vela pulsar, the index is $n=1.4$, which is the smallest one observed.

Actually, some efforts have appeared to find unusual mechanisms to understand the observed braking index. It was suggested previously that the observation of $n \neq 3$ may result from a force-free precession of a distorted neutron star (Macy 1974) or the existence of a companion star (Deminanski \& Proszynski 1979). Peng et al. (1982) and Huang et al. (1982) suggested that neutrino and photon radiation from superfluid neutron vortexes may contribute to pulsar spindown. However, this idea may have difficulty in interpreting $n<3$ since this mechanism dominates for older pulsars with longer periods. Blandford \& Romani (1988) interpreted the observed index by a multipole field and/or field evolution. Melatos (1997) accounted for the spin-down of three pulsars (the Crab, PSR B0540-69, and PSR B1509-58) in the light of a non-standard vacuum dipole model, where a "vacuum radius" is introduced phenomenologically. Recently, accretion torque has been suggested to explain the discrepancy (Morley 1993; Menou et al. 2001). Alpar et al. (2002) proposed that both the usual magnetodipole radiation and the propeller torque applied by the debris disk formed soon after supernova explosion should cause a pulsar to spin down (see also, e.g., Marsden et al. 2001), although no direct evidence exists to show that a normal pulsar has significant torque of this kind.

Send offprint requests to: $\mathrm{R}$. X. Xu, e-mail: rxxu@bac.pku.edu.cn
An alternative effort, within the framework of "standard" neutron stars and their magnetospheric emission models, was proposed by Xu \& Qiao (2001; hereafter Paper I). They addressed that the magnetodipole radiation and the unipolar generator are two different sources powering a pulsar, and these two should be combined in studying pulsar spindown. They find that the calculated braking indices, although modeldependent, are less than 3, and that a proposed model should be ruled out if it cannot result in an index as small as 1.4.

In addition, the pulsar emission mechanism is still a great challenge even over 30 years after discovery. It is a common point that two classes of accelerators (i.e., the inner gaps and the outer gaps) may work on pulsar magnetosphere to reproduce its photon (radio to X-ray or $\gamma$-ray bands) emission, and two more subclasses of models, i.e., the space-charge-limited flow models (e.g., Arons \& Scharlemenn 1979; Harding \& Muslimov 1998) and the vacuum gap models (e.g., Ruderman \& Sutherland 1975) for the inner accelerators appear in the literature. However, it is quite possible that both inner and outer gaps may coexist in a magnetosphere (Usov 2000; Paper I) since those two gaps may work in different field line regions in order to close the global electric current (e.g., Holloway 1975).

In this paper, using the method developed in Paper I, we calculate the braking indices in the models where both inner and outer accelerators lose the rotation energy, neglecting the possible interaction between those two kinds of gaps. Since a new polar gap model, the near threshold vacuum gap (NTVG; Gil \& Mitra 2001) model, became available after Paper I was published, we will begin with computing the braking indices in this new model. It is found that the NTVG model can reproduce an index as small as 1.4, and this model is therefore possible to explain the 5 braking indices observed. However, if the inner and the outer gaps coexist, we suggest that the ICS-induced 
vacuum gap and the outer gap may work together at the same time, since the calculated braking indices in this case are more reasonable than that in other cases.

\section{Braking indices for the NTVG model}

The model of Ruderman \& Sutherland (1975) is "user friendly", but has two imperfect points: 1, the binding energy problem of ions on the neutron star surface; 2, only half of the neutron stars are applicable (e.g., Xu et al. 1999). Although these points can be overcome if some radio pulsars are bare strange stars (Xu et al. 1999), Gil \& Mitra (2001) argued that the RS-type vacuum gaps could also exist for neutron stars with multipolar surface magnetic fields (i.e., the actual surface strength is much higher than the dipolar surface component, and the radius of field curvature is much smaller than the neutron star radius), since the ion cohesive energy becomes larger if the field is stronger.

However, in a superstrong surface field, $B_{\mathrm{s}}>0.1 B_{\mathrm{q}} \simeq$ $4.4 \times 10^{12} \mathrm{G}$, the $\gamma$ photons should be converted into $e^{ \pm}$ at or near the kinematic threshold (Gil \& Mitra 2001); the vacuum gap formed under this condition is thus called as the Near Threshold Vacuum Gap (NTVG) model. Gil \& Melikidze (2002) present the gap heights and the gap potential drops for different pair-production mechanisms (CR-NTVG: curvature-radiation induced NTVG; ICS-NTVG: inverse-Compton-scattering induced NTVG) and different estimates of the cohesive energies of surface iron ions (i.e., Abrahams \& Shapiro 1991, hereafter AS91; and Jones 1986, hereafter J86). In this section, we calculate the braking index for the NTVG model in the frame proposed in Paper I.

For pulsars with given pulsar radius $R$ and polar magnetic field at surface $B$, the braking index $n$ can be written as

$n=3+\frac{\Omega \dot{\eta}}{\dot{\Omega} \eta}=3+\frac{\Omega}{\eta} \frac{\mathrm{d} \eta}{\mathrm{d} \Omega}$,

with

$\eta \simeq \sin ^{2} \alpha+5.4 \times 10^{-9} R_{6}^{-3} B_{12}^{-1} \cos ^{2} \alpha \Omega^{-2} \Delta \phi$,

where $\alpha$ is the inclination angle between the magnetic axis and the rotating axis, $R_{6}=R /\left(10^{6} \mathrm{~cm}\right), B_{12}=B /\left(10^{12} \mathrm{G}\right)$, and $\Delta \phi$ is the gap potential drop which is model dependent.

For the curvature-induced NTVG model, the gap potential drop is (Gil \& Melikidze 2002)

$\Delta \phi_{\mathrm{CR}}^{\mathrm{NTVG}}=4.0 \times 10^{9} \zeta^{1 / 7} \rho_{6}^{4 / 7} b^{1 / 7} P^{-1 / 14} \dot{P}_{-15}^{1 / 14} \mathrm{cgse}$,

with the parameter $b$ in the form

$b_{\text {min }}^{\mathrm{CR}}=\beta \zeta^{0.08} k^{0.57} \rho_{6}^{0.32} P^{-1.15} \dot{P}_{-15}^{-0.5}$,

where $\beta=52$ for AS91 case and $\beta=1990$ for J86 case, $\zeta \sim 0.85$ is the typical value of the correction factor, $k$ is the heat flow coefficient, $P=2 \pi / \Omega$ is the pulsar period in seconds, and $\dot{P}_{-15}=\dot{P} / 10^{-15}$. For simplicity, one may take $k=1.0$ and $\rho_{6}=1$, and therefore has

$\eta_{\mathrm{CR}}^{\mathrm{NTVG}} \simeq \sin ^{2} \alpha+13.7 \beta^{0.14} R_{6}^{-2.38} B_{12}^{-1} \cos ^{2} \alpha \Omega^{-1.76}$.
For the inverse-Compton-scattering-induced NTVG model, the gap potential drop is (Gil \& Melikidze 2002)

$\Delta \phi_{\text {ICS }}^{\mathrm{NTVG}}=1.7 \times 10^{10} \zeta^{0.72} k^{-0.14} \rho_{6}^{1.14} b^{-1} P^{-1.22} \dot{P}_{-15}^{-0.5} \mathrm{cgse}$,

with

$b_{\min }^{\mathrm{ICS}}=\gamma \zeta^{0.25} k^{0.34} \rho_{6}^{0.39} P-1.1 \dot{P}_{-15}^{-0.5}$,

where $\gamma=14$ for AS91 case and $\gamma=130$ for J86 case. One thus has

$\eta_{\mathrm{ICS}}^{\mathrm{NTVG}} \simeq \sin ^{2} \alpha+69.6 \gamma^{-1} R_{6}^{2.25} B_{12}^{-1} \cos ^{2} \alpha \Omega^{-1.88}$.

According to Eqs. (1), (3) and (5), we have calculated the braking indices in different NTVG models, as functions of rotation period for typical pulsars with $R_{6}=1$ and $B_{12}=10$. The results are shown in Fig. 1. It is obvious that the braking index could be smaller than 3 for any inclination angle in the NTVG model. We find all these models can have a braking index as small as 1.4 .

\section{Braking indices in emission models where the inner and outer gaps coexist}

We simply consider the energy conservation between the spindown power and the "dipole radiation + inner gap + outer gap" energy losses, but ignore the interaction between these two kinds of gaps. We expect that this simplified case will still be useful when more detailed and practical consideration is possible in the future. First, however, we correct the calculation of braking index ${ }^{1}$ for the outer gap (Cheng Ho \& Ruderman 1986; Zhang \& Cheng 1997) in Paper I, then we present the results calculated for models where the inner and outer gaps coexist.

For a self-sustaining outer gap, which is limited by the $e^{ \pm}$ pair produced by collisions between high-energy photons from the gap and soft X-rays resulting from the surface heating by the backflowing primary $e^{ \pm}$pairs, the potential drop is (Zhang \& Cheng 1997)

$\Delta \phi^{\mathrm{OG}}=1.6 \times 10^{12} R_{6}^{3} B_{12}^{-1 / 7} \Omega^{-10 / 21} \mathrm{cgse}$,

and the electric current through the gap

$F=8.68 \times 10^{20} B_{12}^{3 / 7} \Omega^{16 / 21}$ cgse,

thus the energy loss rate due to the outer gap could be written as

$\dot{E}^{\mathrm{OG}}=-\Delta \phi^{\mathrm{OG}} F=-1.39 \times 10^{33} R_{6}^{3} B_{12}^{2 / 7} \Omega^{2 / 7} \mathrm{erg}$.

In the case where the outer gap engine dominates (i.e., the inclination angle $\alpha=0$, and only the outer gap works), $\dot{\Omega} \sim$ $\dot{E}^{\mathrm{OG}} / \Omega \sim \Omega^{-5 / 7}$; we have therefore a braking index $n=-5 / 7$ for the outer gap. According to energy conservation, we obtain the $\eta$ value in a model where inner and outer gaps coexist,

$$
\begin{aligned}
\eta^{\mathrm{IG}+\mathrm{OG}}= & \sin ^{2} \alpha+\left(5.4 \times 10^{-9} R_{6}^{-3} B_{12}^{-1} \Omega^{-2} \Delta \phi^{\mathrm{IG}}\right. \\
& \left.+2.25 \times 10^{5} R_{6}^{-3} B_{12}^{-12 / 7} \Omega^{-26 / 7}\right) \cos ^{2} \alpha,
\end{aligned}
$$

\footnotetext{
1 Xu \& Qiao (2001) made a mistake in calculating the braking index for the outer gap, by letting the gap fractional size $f=1$ when writing the particle current.
} 

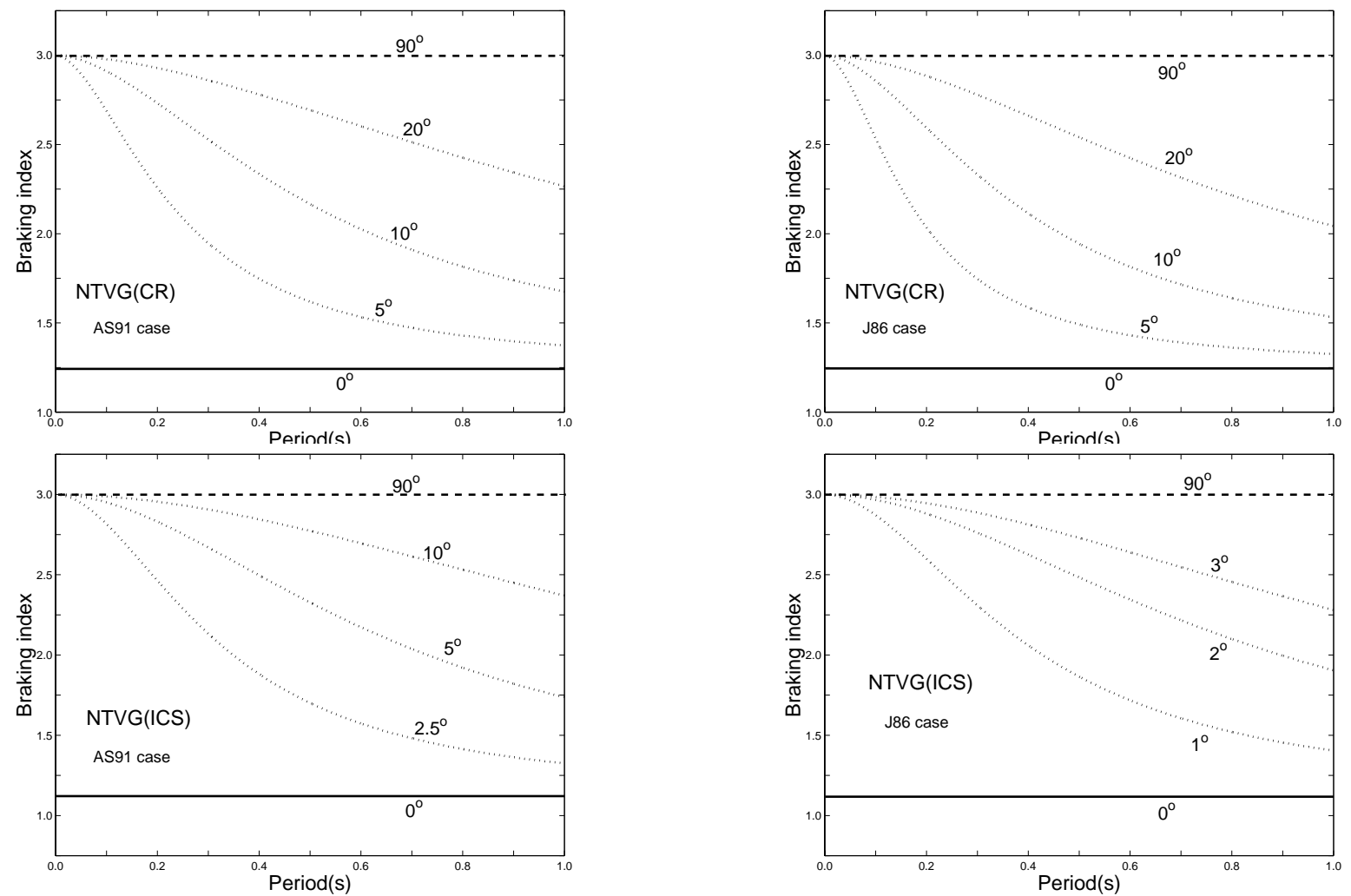

Fig. 1. Set of calculated braking indices, as functions of rotation period, for NTVG models. Pulsars are assumed to have polar magnetic field $B_{12}=10$ and radius $R=10^{6} \mathrm{~cm}$ here. The curves presented are for pulsars with different inclination angles, as indicated in the figure.

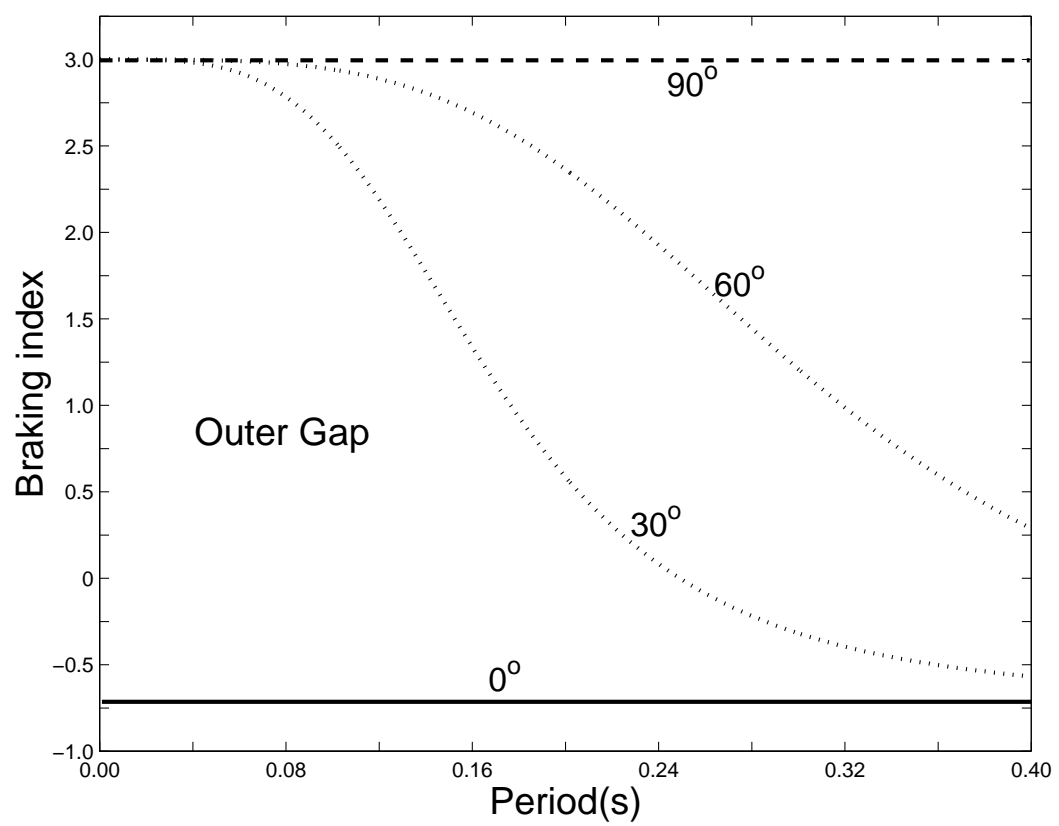

Fig. 2. The braking index calculated in the outer gap model. See Fig. 1 for other notes.

where $\Delta \phi^{\mathrm{IG}}$ is the potential drop of the inner gap. We calculate the braking index, as a function of $P$, from Eq. (1) and Eq. (9). In order to present the correction to Paper I, the new braking index calculated for the case where only the outer gap works is shown in Fig. 2.

In the following subsections, we calculate the braking indices in the inner-outer gap coexisting models. Three kinds of inner gaps are considered, i.e., the normal vacuum gap, the near threshold vacuum gap, and the space-charge-limited flow.

\subsection{Normal VG gap + outer gap}

Various inner accelerator models have been investigated by Zhang et al. (2000). Here we present only the final results of the 

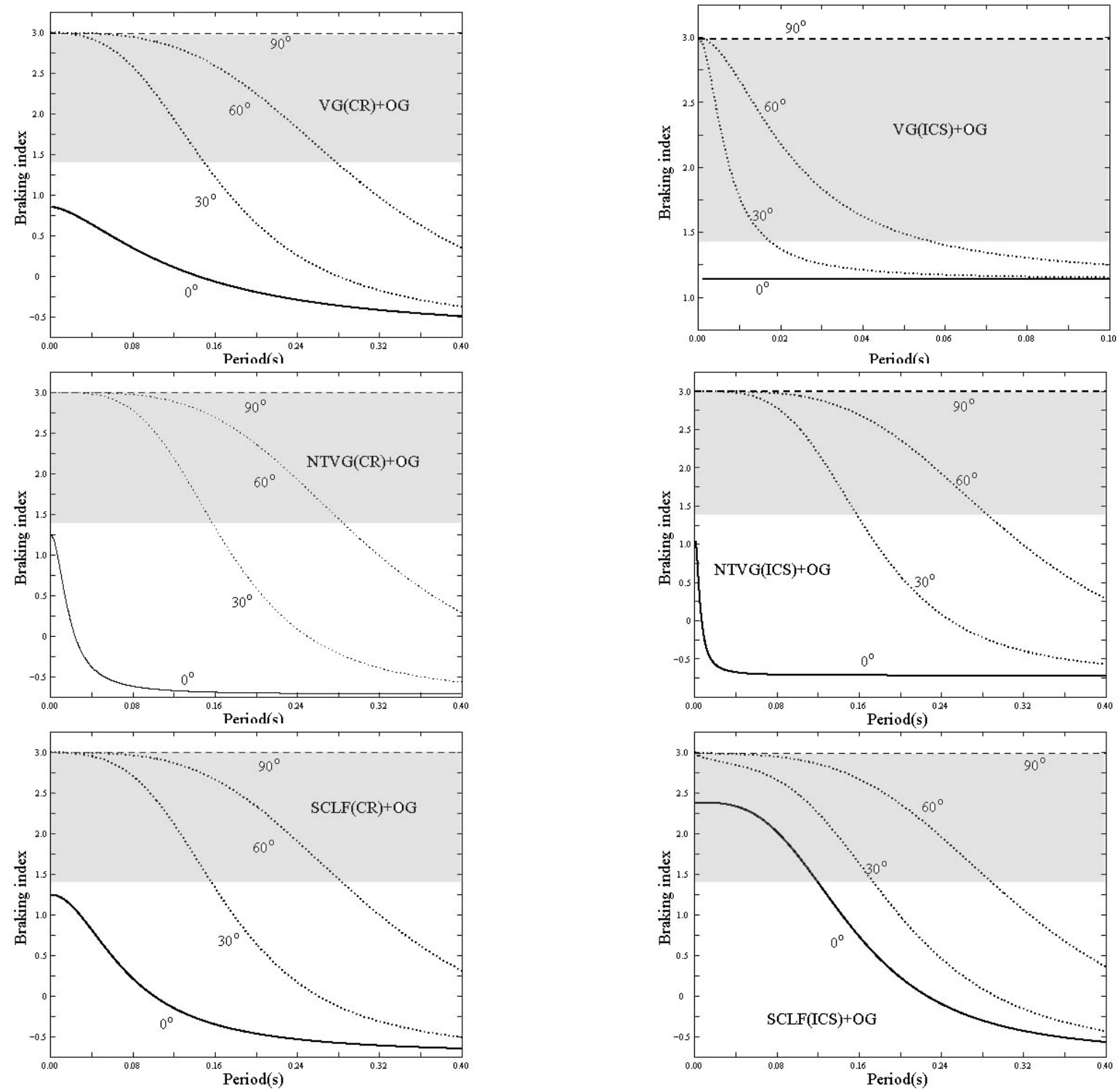

Fig. 3. The braking indices calculated in the inner-outer gap models. The fields are chosen typically as $B_{12}=1$ except for the NTVG models for which we have $B_{12}=10$. The shadowed regions are for observed indices from 2.91 to 1.4. See Fig. 1 for other notes.

$\eta$ parameters for the related models. Details of the gap potentials can be found in Xu \& Qiao (2001) and references therein. For the CR-induced VG case,

$$
\begin{aligned}
\eta_{\mathrm{CR}}^{\mathrm{VG}+\mathrm{OG}}= & \sin ^{2} \alpha+\left(4.97 \times 10^{2} \Omega^{-15 / 7}\right. \\
& \left.+2.25 \times 10^{5} \Omega^{-26 / 7}\right) \cos ^{2} \alpha .
\end{aligned}
$$

For the ICS-induced VG case,

$$
\begin{aligned}
\eta_{\mathrm{ICS}}^{\mathrm{VG}+\mathrm{OG}}= & \sin ^{2} \alpha+\left(1.03 \times 10^{5} \Omega^{-13 / 7}\right. \\
& \left.+2.25 \times 10^{5} \Omega^{-26 / 7}\right) \cos ^{2} \alpha .
\end{aligned}
$$

\subsection{NTVG gap + outer gap}

For the CR-induced NTVG case,

$$
\begin{aligned}
\eta_{\mathrm{CR}}^{\mathrm{NTVG}+\mathrm{OG}}= & \sin ^{2} \alpha+\left(1.37 \beta^{0.14} \Omega^{-1.76}\right. \\
& \left.+2.25 \times 10^{5} \Omega^{-26 / 7}\right) \cos ^{2} \alpha .
\end{aligned}
$$

For the ICS-induced NTVG case,

$$
\begin{aligned}
\eta_{\mathrm{ICS}}^{\mathrm{NTVG}+\mathrm{OG}}= & \sin ^{2} \alpha+\left(6.96 \gamma^{-1} \times 10^{2} \Omega^{-1.88}\right. \\
& \left.+2.25 \times 10^{5} \Omega^{-26 / 7}\right) \cos ^{2} \alpha .
\end{aligned}
$$

\subsection{SCLF + outer gap}

For the CR-induced SCLF case,

$$
\begin{aligned}
\eta_{\mathrm{CR}}^{\mathrm{SCLF}+\mathrm{OG}}= & \sin ^{2} \alpha+\left(38.3 \Omega^{-7 / 4}\right. \\
& \left.+2.25 \times 10^{5} \Omega^{-26 / 7}\right) \cos ^{2} \alpha .
\end{aligned}
$$

For the ICS-induced SCLF case,

$$
\begin{aligned}
\eta_{\mathrm{ICS}}^{\mathrm{SCLF}+\mathrm{OG}}= & \sin ^{2} \alpha+\left(2.27 \Omega^{-8 / 13}\right. \\
& \left.+2.25 \times 10^{5} \Omega^{-26 / 7}\right) \cos ^{2} \alpha .
\end{aligned}
$$

The corresponding braking indices can be calculated from (12) Eq.(1) and one of Eqs. (10)-(15). Figure 3 shows the braking 
index curves, as functions of pulsar periods. It seems that, except for the VG(ICS)+OG model, the energy loss of the outer gap dominates.

\section{Conclusion and discussion}

The braking index in different NTVG models are calculated. The minimum braking indices, $n_{\mathrm{CR}}^{\mathrm{NTVG}}\left(\alpha=0^{\circ}\right)=1.24$ and $n_{\mathrm{ICS}}^{\mathrm{NTVG}}\left(\alpha=0^{\circ}\right)=1.12$, are smaller than 1.4 , the smallest one observed. This indicates that the NTVG model passes the test proposed by Xu \& Qiao (2001), and that this model may work for radio pulsars. In the calculation, typical parameters, e.g., $k=\rho_{6}=R_{6}=B_{12}=1$ (except $B_{12}=10$ in NTVG models), are employed. Nonetheless, we find that the general result does not change significantly if these parameters are adjusted reasonably.

It is suggested that the "ICS-VG + Outer" gaps prefer to work in the pulsar magnetospheres, since other inner-outer gap models imply very small (even a negative) value of the braking index, while the observed indices are in the range from 1.4 to 2.9. These other models may work if much smaller braking indices are observed. For NTVG models, there is little difference between the CR-induced and the ICS-induced ones, because the energy loss rate from NTVG is relatively small compared with that of the outer gap.

Acknowledgements. This work is supported by National Nature Sciences Foundation of China (10273001), by the Special Funds for Major State Basic Research Projects of China (G2000077602), by Chinese Undergraduate Research Endowment in Peking University, and by the Grant 2 P03D 00819 of the Polish State Committee for Scientific Research. JG would like to acknowledge the great hospitality of the Department of Astronomy of Peking University, where this work was started during his visit. RXX thanks Prof. G.J. Qiao for his valuable general discussions about pulsars.

\section{References}

Abrahams, A. M., \& Shapiro, S. L. 1991, ApJ, 374, 652

Alpar, M. A., Ankay, A., \& Yazgan, E. 2002, ApJ, 557, L61

Arons, J., \& Scharlemenn, E. T. 1979, ApJ, 231, 854

Blandford, R. D., \& Romani, R. 1988, MNRAS, 234, 57

Cheng, K. S, Ho, C., \& Ruderman, M. 1986, ApJ, 300, 500

Deminanski, M., \& Proszynski, M. 1979, Nature, 282, 383

Gil, J., \& Melikidze, G. I. 2002, ApJ, 577, 909

Gil, J., \& Mitra, D. 2001, ApJ, 550, 383

Harding, A. K., \& Muslimov, A. G. 1998, ApJ, 508, 328

Holloway, N. J. 1975, MNRAS, 171, 619

Huang, J. H., Lingenfelter, R. E., Peng, Q. H., \& Huang, K. L. 1982, A\&A, 113, 9

Jones, P. B. 1986, MNRAS, 218, 477

Macy, W. W. Jr 1974, ApJ, 190, 153

Marsden, D., Lingenfflter R. E., \& Rothschild R. E. 2001 ApJ, 547, L45

Melatos, A. 1997, MNRAS, 288, 1049

Menou, K., Perna, R., \& Hernquist, L. 2001, ApJ, 554, L63

Morley, P. 1993, e-print [astro-ph/9311036]

Peng, Q. H., Huang, K. L., \& Huang, J. H. 1982, A\&A, 107, 258

Ruderman, M. A., \& Sutherland, P. G. 1975, ApJ, 196, 51

Usov, V. V. 2000, in IAU Colloq. 177, Pulsar Astronomy-2000 and Beyand, ed. M. Kramer, N. Wex, \& R. Wielebinski (San Francisco: ASP), ASP Conf. Ser., 202, 417

Xu, R. X., \& Qiao, G. J. 2001, ApJ, 561, L85 (Paper I)

Xu, R. X., Qiao, G. J., \& Zhang, B. 1999, ApJ, 522, L109

Zhang, B., Harding, A. K., \& Muslimov, A. G. 2000, ApJ, 531, L51

Zhang, L., \& Cheng, K. S. 1997, ApJ, 487, 370 